Intersections

Canadian Journal of Music

Revue canadienne de musique
Intersections

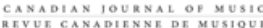

\title{
Michel Chion, théoricien, musicien et cinéaste
} Interview

Volume 33, numéro 1, fall 2012

URI : https://id.erudit.org/iderudit/1025555ar

DOI : https://doi.org/10.7202/1025555ar

Aller au sommaire du numéro

\section{Éditeur(s)}

Canadian University Music Society / Société de musique des universités canadiennes

ISSN

1911-0146 (imprimé)

1918-512X (numérique)

Découvrir la revue

Citer ce document

(2012). Michel Chion, théoricien, musicien et cinéaste : interview. Intersections, 33(1), 51-64. https://doi.org/10.7202/1025555ar

Copyright @ Canadian University Music Society / Société de musique des universités canadiennes, 2014
Ce document est protégé par la loi sur le droit d'auteur. L’utilisation des services d’Érudit (y compris la reproduction) est assujettie à sa politique d'utilisation que vous pouvez consulter en ligne.

https://apropos.erudit.org/fr/usagers/politique-dutilisation/ 


\section{MICHEL CHION, THÉORICIEN, MUSICIEN ET CINÉASTE}

\section{L'UTILISATION DE LA MUSIQUE CONCRÈTE AU CINÉMA}

Michel Chion: Je précise tout d'abord que j'emploie depuis plus de vingt ans l'appellation de «musique concrète» dans le sens de "musique de sons fixés", quelles que soient les techniques employées pour faire les sons, la source initiale de ceux-ci, les machines employées, et l'esthétique. L'appellation «musique concrète» a été inventée et définie (dans des termes très proches) par Pierre Schaeffer en 1948, mais c'est en 1988 que j'ai proposé l'expression de «son fixé», plus pertinente selon moi que celle de «sons enregistrés», pour définir le genre comme un "art des sons fixés». J'ai bien dit "pour le définir», non pour le nommer, puisque son nom est musique concrète.

Dans le cadre de cette définition du genre (qui englobe ce que selon les époques et les pays on appelle ou on a appelé aussi "musique acousmatique», "musique pour bande», et même "musique électroacoustique» lorsque ce terme ne désignait que les musiques sur support), je pense que même si les techniques et les machines sont identiques, composer de la musique concrète sans images, pour le concert, comme je le fais, et composer de la musique concrète en rapport avec l'image et surtout dans le cadre d'un film - ce que j'ai expérimenté dans les années 1980 et que j'ai cessé de faire ensuite, sauf pour mes propres réalisations - , sont deux domaines différents. Avec le cinéma, l'image transforme l'audition du son et le son transforme la vision de l'image, et surtout les péripéties de l'histoire orientent tout ce que l'on perçoit, images et sons.

Je constate toujours que la musique concrète devient autre chose quand on l'entend dans un contexte cinématographique. De nouvelles causes potentielles, qui appartiennent à l'univers figuratif du film, se proposent pour les sons, et se mettent à leur donner de nouvelles significations. C'est une interprétation de la musique, un peu comme peut la faire un ballet. La musique concrète au cinéma entre en rapport non seulement avec l'image, mais avec le scénario, le dialogue et le sens, qui changent tout, et d'autre part, elle est présentée par tronçons dispersés. Par conséquent, sous cette forme, l'œuvre de musique concrète, faite pour être écoutée seule et en tant que totalité, ne peut pas être appréciée pour elle-même.

La musique de film en général, d'ailleurs, même instrumentale, n'est pas faite pour être écoutée seule. Si quelqu'un se sent frustré en faisant de la musique de film ou de la conception sonore, ou en constatant que son travail n'est pas apprécié pour lui-même par le spectateur, ou qu'il n'est pas mis en valeur par 
le mixage et le montage du film, c'est qu'il ne veut pas comprendre comment fonctionne le cinéma.

Mais ce qu'il se passe en plus avec la musique concrète, c'est qu'elle fait souvent entendre des sons de source non instrumentale au sens classique, qui se prêtent dès lors à des associations causales sauvages. Alors, quand on entend de la musique concrète dans un film, quel moyen nous permet de faire la séparation dans le son du film entre ce qui est l'apport du compositeur et les sons diégétiques impliqués dans la narration cinématographique, ambiances, sons mécaniques, rumeurs naturelles, etc.? La différenciation est impossible. C'est, à mon avis, la raison pour laquelle la musique de film continue d'être instrumentale et orchestrale les neuf dixièmes du temps au cinéma: même quand son écriture est atonale, cela permet, par l'identification des timbres instrumentaux, de séparer ce qui relève de la musique faite par un musicien, et ce qui relève des bruits de l'action. Dans les sources instrumentales, j'inclus bien sûr les instruments électroniques à clavier.

Quand George Lucas a réalisé La Guerre des étoiles, en 1977, de nombreux cinéphiles ont été frappés par l'absence de sons électroniques dans la partition de John Williams : "C'est une musique orchestrale, on dirait du Wagner, alors que c'est un film de science-fiction!» Je me suis demandé pourquoi Lucas avait adopté ce parti-pris. C'est peut-être parce que, dans son film, il y a beaucoup de bips bips, de grondements et de vroarrrr réalisés par Ben Burt, un grand artiste des effets sonores, et que Lucas a voulu que ces effets soient perçus par le spectateur comme concrets, figuratifs, étroitement reliés à l'action, aux objets, aux gestes, aux animaux et aux machines qu'on y voit. Plus récemment, on peut citer le film en $3 \mathrm{D}$ de James Cameron, Avatar, où tout est moderne et futuriste, sauf la musique, laquelle est classiquement orchestrale. Peut-être pour ces films est-il plus efficace aux yeux de leurs réalisateurs de créer une séparation absolue de la musique et des effets, de ne pas confondre les éléments de l'action et ceux provenant de la fosse. Il y a bien sûr quelques films de science-fiction qui ne rentrent pas dans ce modèle: par exemple, Planète interdite, en 1956, avec les sons électroniques de Louis et Bebe Barron, ou Blade Runner, la musique de synthétiseur de Vangelis se mélangeant aux effets sonores associés au décor et à l'action (ville futuriste, véhicules individuels volants, etc.). J'aime énormément le film de Ridley Scott, sorti en 1982, mais je constate que cette formule est restée très minoritaire. On peut reprendre ce parti-pris, si l'on veut, mais il ne faudra pas s'étonner si, dans la perception du spectateur, il se produit une sorte de méli-mélo, de confusion pour les sons entre l'action, l'atmosphère et la musique.

Question: Est-ce que l'absorption des meilleurs travaux de musique concrète par les images est une chose réglée et inévitable? Est-ce que toute image, quel que soit son contenu, son cadrage, son montage, va toujours absorber le son, la musique? J'imagine qu'il y a quelques compositeurs de musique concrète dans la salle. Est-ce qu'ils peuvent espérer que, dans tel ou tel complexe d'images et de sons, il y ait une tension à tout le moins entre des combinaisons de sons 
et des images, de sorte que quelque chose arrive qui ne soit pas de l'ordre de l'absorption de l'un par l'autre?

Michel Chion: S'il y a absorption, ce n'est pas celle du son par l'image: c'est celle de l'image et du son par la diégèse, l'histoire - l'image est elle-même absorbée par l'histoire racontée. Pour le cas particulier du son, votre question, qui est très précise, touche entre autres à la distinction que je propose de faire entre ce que j'appelle l'écoute figurative (ce que ça représente, ce que ça figure) et l'écoute causale (avec quoi nous supposons ou nous savons que c'est réellement fait). Ces écoutes peuvent converger, mais aussi diverger. Nous pouvons reconnaître en "écoute figurative» un son de vent fait avec un synthétiseur, même si nous identifions le synthétiseur comme en étant la cause réelle. Par ailleurs, vous pouvez enregistrer des pétillements de feu de bois en approchant le microphone, vous mettez ça sur un support d'enregistrement, vous le faites passer par un haut-parleur, et l'auditeur, à moins d'être informé de la source réelle, ne reconnaîtra pas nécessairement du feu. Il entendra quelque chose qui pétille, mais l'identification figurative de l'auditeur, par rapport à ce que c'est, pourra, dans certains cas, flotter entre la pluie et le feu. Je trouve ça intéressant, parce que symboliquement, l'eau et le feu, ce n'est pas la même chose, tout en étant lié. Je fais ici référence à des passages très précis de mon œuvre de musique concrète La Tentation de Saint Antoine, notamment le début du tableau intitulé Le Nil, où j'utilise cette ambiguité eau-feu (dans l'écoute figurative), en me servant de sons que je n'ai créés ni avec l'eau, ni avec le feu, mais avec des craquements de surface de disque.

Dans l'expérience quotidienne, nous croyons souvent identifier les sons par eux-mêmes, alors que c'est le contexte qui les identifie: quand nous sommes chez nous, les bruits venant de la cuisine, portes de placards, vaisselle qu'on manipule et robinet qui coule - leur cause humaine, nous la reconnaissons, car si ce n'est pas nous qui les actionnons, c'est selon toute vraisemblance la personne avec qui nous occupons l'appartement à ce moment-là. Mais en dehors de ce contexte, cela pourrait être n'importe qui, et parfois n'importe quoi. Ce choc qui vient de l'étage au-dessus peut être une chute d'objet, une chute de personne, accidentelle ou non, comment savoir? C'est ce que j'appelle le flou narratif du son. Les sons, hors de leur contexte et notamment en situation acousmatique, c'est-à-dire quand on ne voit pas leurs sources, permettent beaucoup moins qu'on ne le croit d'identifier une cause précise. Or, au cinéma, vous avez une image figurative, un mur, une porte, etc. et sa figurativité contraint les sons qui ne sont pas reconnus comme instrumentaux au sens classique à prendre un sens précis. 99\% du cinéma est figuratif, pas forcément narratif, mais figuratif, et quand il est narratif, il est ipso facto figuratif aussi, c'est-à-dire qu'il représente des choses. L'image elle aussi est assujettie à cet aspect figuratif et narratif.

Lattention du public va être conditionnée par la cause qu'il croit identifier du son à travers l'histoire racontée. Un son qui est un son, en musique concrète, devient, dans le cadre d'un film, le son d'un vaisseau spatial, d'une machine, d'un objet précis. Et, du coup, il perd le flottement que j'évoquais. Comme je 
l'ai dit, je crois que c'est pour cette raison que l'on continue d'utiliser la musique instrumentale.

Je pense que la recherche de l'identification causale du son est incontournable pour l'être humain. Il a besoin de savoir avec quoi c'est fait, ou de pouvoir en avoir une idée. Pourquoi? Parce que le son est, dans notre expérience animale et humaine, quelque chose qui peut nous avertir d'un danger ou d'un problème. Il est inutile de dire à l'auditeur de ma musique: «Ne pensez plus aux causes». Je peux lui dire: «Laissez flotter les causes, les associations. Faitesmoi confiance, personne n'a été égorgé pour faire les sons que vous entendez dans cette musique concrète. " Même si la cause réelle est identifiée, l'écoute figurative continue de flotter. Lorsque je regarde une toile du peintre Jackson Pollock, je vois des nébuleuses et des constellations. Je sais que ce n'est pas de la peinture figurative, peu importe, mais je n'identifie pas ce que je vois à une figuration précise, je laisse flotter mes associations. Je ne dis pas «la musique représente ceci», "ce tableau représente cela». Il faut laisser flotter.

C'est d'ailleurs ce qu'invite à faire ma musique concrète: elle coupe le cordon entre écoute causale et écoute figurative. Dans Le souffle court, créé à Montréal grâce à Réseaux, j'ai enfin incorporé des sons que j’avais faits en 1970 et qui n'avaient jamais pu entrer dans une de mes œuvres. J'ai souvent retravaillé ces sons, ils ont traversé plusieurs stades. Au bout de vingt transformations, joublie souvent totalement avec quoi ils ont été faits. Ça devient des sons en soi, indépendants de leur source réelle. C'est une manière de travailler qui me plaît, certes, mais qui est impossible dans un contexte cinématographique. En effet, le cadre audiovisuel figuratif arrête le flottement et fixe l'identification causale. De toute façon, le son d'un film est conçu par couches: les dialogues, les bruits, la musique... Le spectateur cherche à séparer les couches mentalement.

C'est pour cela que je pense que la musique concrète, telle qu'employée au cinéma, se dilue. Elle devient de l'atmosphère ou de l'ambiance vaguement réaliste ou vaguement expressionniste. Elle peut rapporter des droits d'auteur, mais c'est son seul intérêt pour un musicien. Que celui-ci ou celle-ci ne s'étonne pas si son travail, beaucoup plus qu'une musique instrumentale et tonale, est complètement «détourné » par le film.

\section{LA MESSE DE TERRE: RYTHME ET TEXTURE AUDIO-VISUELLE}

Michel Chion: Je voulais faire une messe audio-visuelle, ou plutôt audio-logovisuelle à partir d'éléments du quotidien, qui se mettent à prendre un sens sacré par le côté répétitif et lancinant, comme une psalmodie. J’ai réalisé à la fois le son, l'image et le montage, entre 1991 et 1996. À l'époque où je l'ai commencée, j'emmenais avec moi une petite caméra vidéo Sony de format V8, le format vidéo amateur de l'époque. J'avais filmé l'orage qu'on voit dans cette séquence depuis une voiture que conduisait ma femme Anne-Marie, au cours de vacances dans les Pyrénées, non loin de la maison qu'habitait alors mon père. Je ne savais pas quoi faire de cette prise de vue, mais j'aimais bien son côté fellinien, avec ces gens en maillot de bain qui se dispersent sous l'orage dans une atmosphère de catastrophe. Le son «direct» enregistré avec l'image 
en cassait d'ailleurs l'ambiance, puisqu'on entendait un speaker à la radio, et les dialogues que j'échangeais avec Anne-Marie, et bien sûr, je n'en ai pas utilisé une seule seconde.

Parallèlement, j’avais créé en studio, tout à fait indépendamment, cette séquence sonore qui va avec, et dans laquelle vous entendez sur différentes couches composées des sons électroniques produits sur un synthétiseur et des sons de frottement, créés et enregistrés en studio avec un magnétophone (je frottais un micro contre les plateaux tournants du magnétophone). Donc un système tournant qui générait des rythmes un peu comme une machine qui grince. Et, à un moment donné, dans la réalisation de la Messe, j'ai eu l'idée de faire se rencontrer ces sons et ces images, et je me suis dit: «Je vais voir ce que ça donne si je ralentis l'image de l'orage et si je la mets en parallèle avec ces séquences sonores ». Évidemment, j'ai remonté beaucoup l'image par rapport au son. Dans l'image, ce qui marchait bien, c'est que j'ai plusieurs références temporelles rythmiques. J'ai «l'avancée» de la voiture, j'ai les gens qu’on dépasse, qui marchent, et j'ai l'essuie-glace et son oscillation, j'ai la chute précipitée des gouttes de pluie. Donc, par ralentissement et accélération de ce battement pendulaire, je peux utiliser l'essuie-glace comme un rythme variable très expressif.

Je trouvais que ça marchait, en raison notamment des «lignes de fuite temporelles »: dans ce cas précis, pour l'œil, on est sur une route, on pénètre dans un espace, les gens s'approchent, donc on prévoit à l'avance, tandis que le son dans cette séquence, lui, on l'entend à court terme, on est pris dans une sorte d'éternel présent qui piétine. À l'image, quelque chose s'approche, disparaît, s'approche et disparaît encore, et dans le son il se produit une sorte de marmonnement, de ressassement hypnotique, mais celui-ci, à cause de l'image, semble aller vers un but. Les deux logiques se combinent, je crois, admirablement.

L'identité de texture entre image et son fonctionne également bien. Je suis très attaché dans la création des sons à la texture, à la matière, à une qualité tactile que j'obtiens par toutes sortes de procédés. Je broie les sons, avec ou sans bande magnétique, afin de leur donner cette matière. Dans l'image, j'avais également une texture donnée par la faible définition du format V8, mais aussi par ce qui était filmé: la pluie qui tombe sur le pare-brise est tellement dense qu'elle brouille ce que l'on voit. L'essuie-glace nous donne un coup de balai là-dessus, et ça réapparaît. C'est une micro-texture temporelle, ce que j'appelle les microrythmes (la pluie, les feuilles sous l'effet du vent, etc.). Les deux textures dialoguent bien, et les deux phénomènes audio- et visuels, par leur densité, s'allient, ne font qu'un. C'est un peu comme voir deux danseurs ou, comme chez Steve Reich, où les cycles se déphasent et se re-phasent. Les univers se complètent par le rythme et la texture.

Nous sommes dans un univers polyrythmique. Le son de cette séquence de L'Introît de la Messe de terre comporte quatre ou cinq couches: il y a des sons électroniques, comme des chants d'oiseaux, très irréguliers; le rythme qui est fait avec le magnétophone, et que je varie; ma voix qui psalmodie sur différents rythmes. Dans l'image, vous avez aussi plusieurs rythmes visuels: les micro-rythmes de pluie; le rythme latéral de l'essuie-glace; le rythme en profondeur des gens qui avancent. Le ralentissement de l'image crée des saccades 
(j'ai filmé à vitesse normale, ce n'est pas un ralenti sportif où on filme à 1000 images minute pour voir des ralentis nets et continus, ça a été tourné à 25 images/seconde). Je joue sur ce genre d'éléments. L'effet global est une sorte de progression laborieuse et pénible, comme lorsqu'on monte une pente, que l'on souffre et qu'on veut y arriver. Souvent au début de mes œuvres, cela vaut également pour ma musique concrète, c'est dur de se lever, de s'y mettre, et après ça va mieux.

Je pense que si vous n'avez pas d'éléments de textures ou de micro-rythmes en commun ou en rapport clair entre le son et l'image, l'accrochage se fait plus difficilement. Dans Blade Runner, dont je parlais tout à l'heure, le rapport du son et de l'image est très beau grâce aux nombreux papillotements dans le son, et aux mouvements de fumée et de lumière dans l'image. C'est magnifiquement vivant. D'autres films ont cherché à faire la même chose et, à mon avis, ça n'a peut-être pas marché parce que l'image est tellement nette et précise et fixe que ça ne peut pas s'accrocher. Le son et l'image, il faut que ça s'accroche quelque part, sinon on constate qu'il y a juste cœxistence plate et indifférente de deux univers. Dans Shining, Kubrick aimait bien, au contraire, une lumière très froide, très nette. Mais, à partir du moment où ce film a une image très pure, très géométrique, très symétrique, très éclairée et très fixe, il ne pouvait pas mettre le même type de sons. Les sons ne s'accrochent pas, ils dérapent sur l'image parce qu'il n'y a pas de points d'accrochage. Mais ce dérapage peut d'ailleurs produire des effets très intéressants. Donc, à chaque fois, il faut trouver une solution différente. Je crois qu'il faut être très sensible à cette questionlà, que j'appelle trans-sensorielle, dont les deux grandes dimensions sont le rythme et la texture.

\section{LE RAPPORT DU COMPOSITEUR AVEC SES OUTILS}

Je poursuis mon travail avec le support de la bande magnétique, mais ça m'est personnel. Peu importe la nature du support, c'est de la musique concrète dès lors où une musique s'identifie à ce qu'il y a sur le support. Aujourd'hui, le support en vogue est encore le CD, donc un support qui se copie facilement, qui n'a plus aucune valeur en tant que support, ce qui d'ailleurs est un peu dommage selon moi parce que parfois la nature matérielle, tangible du support, peut aider à valoriser l'œuvre. De cette façon, la pellicule a longtemps aidé à valoriser les films...

Il faut distinguer, pour la musique concrète ou pour toute œuvre basée sur le principe du support, le support de fixation de l'original de l'œuvre terminée, laquelle doit être stable, fidèle et précis, et les supports de travail employés pour le tournage sonore, pour les traitements, le montage et le mixage des sons, qui peuvent être absolument variés et quelconques. Aujourd'hui, dans un film, on peut mélanger du super 8 , de la photographie, du numérique, du dessin, etc. Il n'y a absolument aucune raison de se refuser quoi que ce soit sur le plan des supports de travail. C'est pour ça d'ailleurs que j'aurais aimé qu'en France, dans les studios institutionnels comme le GRM de Paris (dont j'ai démissionné en 1977, tout en y réalisant par la suite, comme compositeur invité, une partie 
de mes musiques concrètes), on conserve les magnétophones, les machines anciennes, parce qu'elles ont des sons, des possibilités, des propriétés particulières. Gardons tout ! D’autant d'ailleurs que la quasi-totalité du répertoire de la musique concrète d'avant les années 1980 - à part ses toutes premières œuvres - a été réalisée sur bande magnétique et qu’une œuvre a, sur son support d'origine, toutes les chances d'être entendue à son meilleur. Malheureusement, les studios français et d'autres dans le monde n'ont pas voulu ou pu conserver ces machines. Mais il y a des milliers de magnétophones à bandes qui attendent dans les remises et les sous-sols. J'encouragerais les gens à les récupérer, car ce sont des appareils précieux qui, en outre, je le constate en France, sont de plus en plus recherchés par de jeunes musiciens. De même, il existe des milliers de bobines de bande magnétique dans le placard des radios ou des centres de musique électroacoustique. Une bande magnétique, ça vit assez longtemps, ça se réutilise, ça s'efface, ça se réemploie; c'est ce que je fais avec les miennes. Même si les laboratoires et les compagnies ont arrêté d'en fabriquer, il y a assez de bandes sur la planète pour les cent prochaines années. Quant aux techniques pour exploiter ces appareils, je les ai décrites à l'usage des personnes intéressées dans plusieurs articles qu'a publiés la revue française Revue \& Corrigée (articles repris en annexe dans la nouvelle édition de mon livre La Musique concrète, art des sons fixés).

J'aime le magnétophone pas seulement parce qu'il permet de jouer les œuvres des années 1950 à 1980 dans des conditions optimales. D’abord, la bande magnétique donne des possibilités de transformation du son que ne donne pas l'ordinateur et vice versa. Pourquoi alors ne pas avoir les deux? Je ne vois pas pourquoi, en peinture, quelqu'un dirait: "Je ne vais pas utiliser ce crayon-là parce qu'il appartient aux années $1950 \%$. De nombreux photographes continuent d'employer la pellicule et des vieux objectifs, ou le procédé Polaroid de développement instantané. Et, troisième raison (il y en a encore bien d'autres), j'aime travailler sur magnétophone parce que j'aime toucher le support. Vous me direz que c'est purement personnel, oui, mais ça peut compter. Quelqu'un peut très bien s'ennuyer profondément devant un écran d'ordinateur à promener sa souris et se trouver content de rembobiner la bande, d'y toucher, de la regarder tourner.

C'est valable pour d'autres domaines de l'art. Mon amie Christiane Sacco avait réussi à écrire son premier roman parce qu'elle tapait à la machine ellemême ses premiers jets. Elle n'était pas une dactylo professionnelle, comme on disait à l'époque, mais la machine à écrire, électrique ou électronique, lui donnait une sorte de dynamisme. Dès lors où nous sommes passés au traitement de texte, elle a eu beaucoup plus de mal à écrire, et je lui ai proposé de taper ses textes pour l'aider. Elle habitait à Lyon, moi à Paris, et elle m'envoyait ses brouillons écrits à la main, je les tapais et les lui renvoyais, mais je voyais bien qu'elle avait perdu son élan. Cela ne lui suffisait pas de griffonner des pages à la main, et ensuite de faire des corrections manuscrites sur les pages que je tapais et imprimais. Comment dire... elle a été brisée dans les petites choses qui faisaient qu'elle avait du dynamisme à écrire, que ce n'était pas seulement une corvée. Et je pense que ça compte. 
Le réalisateur suédois Ingmar Bergman racontait que lorsqu'il écrivait un scénario, il avait besoin d'écrire à la main et d'utiliser des petits cahiers à carreaux. Puisqu'il était prévoyant et très bien organisé, et aussi parce qu'il avait peur qu'un jour on arrête de fabriquer ces petits cahiers à carreaux, il en a acheté toute une caisse. Il les a mis dans sa maison, sur une île, puis toute sa vie il a travaillé sur ces petits cahiers à carreaux pour écrire ses scénarios.

Il est parfois difficile de trouver ou de faire réparer un magnétophone selon le pays ou le lieu où l'on se trouve. Mais grâce à Internet, on peut trouver à présent des adresses utiles à cette fin. Cette dimension-là, c'est-à-dire le bon rapport physique entre l'artiste et ses moyens, me semble très importante. Chacun a droit à une subjectivité totale dans le choix de ses moyens: il peut écrire au crayon, à la machine à écrire mécanique, sur l'ordinateur ou sur une tablette bien sûr, sur tout ce qu'il veut, si c'est le bon moyen pour lui. On est là dans le monde concret : écrire en écriture manuscrite sur une tablette avec un stylet, et écrire sur du papier avec un stylo ou un crayon sont deux expériences profondément différentes, on peut aimer l'une et l'autre, l'une et pas l'autre, aucune des deux. On pourrait aussi, pour rester dans le domaine de l'écriture, dicter à une autre personne (c'est, du reste, de cette manière qu'ont été faits beaucoup de livres autrefois) ou à un système de reconnaissance vocale, comme c'est possible de le faire de nos jours. Mais ne laissez personne vous imposer des moyens de travail avec lesquels vous ne vous sentez pas à l'aise sous prétexte que «c'est mieux» ou plus «moderne».

Des outils ont également multiplié les possibilités sonores. En 1988, j’ai inventé un type de manipulations avec un magnétophone que j'ai baptisé «crayonné»; personne n'avait eu l'idée d'employer cet appareil depuis son invention, une quarantaine d'année auparavant. Ce procédé permet la réalisation de montages et de mixages de façon nouvelle, avec un résultat radicalement différent. Je le décris dans La musique concrète, art des sons fixés.

L'un des avantages que présente le magnétophone, du moins pour certaines étapes de travail, est que je ne suis pas obligé de regarder quelque chose. J'aime bien pouvoir entendre le son sans support visuel graphique, lequel fait semblant de vous montrer ce que vous entendez alors que ça n’a rien à voir. L'ordinateur m'oblige à fixer des yeux un écran.

Une autre chose positive, c'est que le magnétophone me permet de travailler debout. Avec l'ordinateur, je peux aussi me mettre debout, mais le fait d'avoir un écran, d'avoir la tête dirigée quelque part ne me convient pas par rapport à ma manière de travailler. Vous voyez, ce sont des choses très pragmatiques.

Question: Est-ce que vous êtes du genre à trafiquer le magnétophone ou à respecter l'outil? Est-ce que vous respectez les déterminations techniques de l'outil, ou vous essayez de les transformer?

Michel Chion: Tout dépend de ce que l'on entend par «trafiquer». Forcément, je ne vais pas casser l'appareil, parce que celui-ci, soit ne m'appartient pas, soit, quand il m'appartient, n'est pas si facile à réparer ou à remplacer. Mais la manipulation baptisée "crayonné» suppose, par exemple, des gestes avec la bande magnétique qui ne sont pas prévus, même s'ils n'obligent pas à modifier 
la mécanique du magnétophone, et s'ils n'ont aucun effet destructeur sur l'appareil.

Dès le début, j'ai souvent fait des sons qui, par rapport aux recommandations techniques de ce qu'on appelle la "haute-fidélité», étaient jugés mauvais et saturés. Quand j'ai réalisé le Requiem, en 1972-1973, donc à l'âge de 25 ans, j'appartenais encore au Groupe de recherches musicales (le GRM), et on m'a fait à l'intérieur du Groupe beaucoup de reproches techniques à propos de cette œuvre. "Certains de tes sons sont saturés», me disait-on alors. Il ne fallait pas, justement, qu'ils soient saturés. «Il y a du souffle», me reprochaiton encore. C'était une époque où l'on pensait que le pire était d'entendre le souffle de la bande magnétique. Le souffle, tout le monde s'en fiche quand c'est intéressant, quand c'est vivant. Je ne dis pas qu'il donne à lui tout seul de la chaleur au son, je ne suis pas non plus fétichiste du bruit de fond de la bande magnétique. Mais je constate que si l'on cherche à l'éliminer ou à l'éviter à tout prix, parfois les sons sont moins intéressants. Dans le Requiem, on m'a aussi dit, à l'époque, qu'on entendait les plops du micro, les chocs de la respiration, ou d'une consonne plosive comme le «p». Évidemment, c'était intentionnel. Cela participe pour moi d'une dramatisation propre au son fixé, de la même façon qu'avec les instruments classiques, on peut créer une dramatisation du son instrumental ou vocal (utilisation des registres extrêmes, de la virtuosité, des timbres décolorés, etc.).

C'est comme dans un film: si vous voyez fugitivement le reflet du soleil ou d'un phare de voiture sur une lentille de l'objectif de la caméra, ou si vous ressentez l'instabilité de la caméra qui filme. Ça vous gêne? Moi ça ne me gêne pas, à partir du moment où ça contribue à la vie du film. Il y a longtemps que ça ne fait plus problème au cinéma, alors que pour cette musique-là, il y a encore des gens qui pensent qu'il faut la faire comme si l'on devait faire oublier aux gens qu'elle sort de haut-parleurs, qu'elle a été faite avec des appareils enregistreurs, des micros, un support, etc. Aujourd'hui encore, beaucoup de compositeurs font comme s'il fallait qu'on cache que les sons sont montés, donc ils vont faire des transitions sonores bien étudiées pour que tout ait l'air de se dérouler naturellement et d'être fait indépendamment du support (ce que j'appelle l'esthétique «naturaliste»). Tout cela révèle la peur de laisser apparaître que c'est du son fixé. C’est pour cela que j'ai proposé, pour la musique concrète, la définition art des sons fixés: pour que soit enfin assumée cette dimension.

Dans les années 1960 et 1970, j'ai participé en tant que chanteur choriste à des enregistrements d'ouvres chorales avec orchestre. J'ai découvert que l'on enregistrait une œuvre classique par petits bouts. Les quatre premières pages, les cinq premières, et ensuite la reprise ; puis le montage des bonnes prises... comme pour un film. Mais les gens qui achètent les disques classiques ne veulent pas savoir qu'il y a du montage et que le grand chef d'orchestre a enregistré la symphonie de Mahler par fragments de cinq à dix minutes. Sur le livret du disque, il n'y est nulle part fait mention de ce montage, pourtant bien présent. 


\section{LES IDÉES STRUCTURANTES DU SON ET L'IMAGE}

Question: Pensez-vous que les mêmes idées structurantes (que Pierre Schaeffer a lui aussi tenté de formuler) pour la musique concrète pourraient s'appliquer, d'une façon ou d'une autre, à la création d'une imagerie visuelle abstraite, laquelle serait le pendant de la musique concrète?

Michel Chion: C'est une bonne question, que Schaeffer s'est d'ailleurs posé luimême. Je pense en fait qu'une partie de ce travail de création et de description pour le visuel existe déjà depuis des siècles. Quand on perçoit la forme d'un cercle et qu'on reconnaît cette forme dans une assiette, dans le soleil ou dans certaines fleurs, on produit ce genre d'association. On est habitué, pour le visuel, à abstraire les formes des objets, tout en reconnaissant la nature de ces objets : assiette, fleur, soleil. C'est ce que Schaeffer a cherché à faire avec son solfège des objets sonores, et je continue largement son travail, notamment dans mon ouvrage Le son, traité d'acoulogie.

Alors comment faire écouter des objets temporels évolutifs? Certains sons sont stationnaires, oui, mais ceux-là sont plutôt rares, à part peut-être ceux fabriqués par l'homme à partir de machines. Auquel cas, les sons sont évolutifs. Le son peut donc plus difficilement s'arracher au temps. On a pu en rêver, mais on ne peut pas le faire parce que ce qui définit le son est lié à son évolution dans le temps, même un son de piano. Donc, comment étudier les objets sonores dans le temps? Schaeffer a eu l'idée de privilégier des objets pas trop longs, qui peuvent servir de question spécifique au domaine sonore, qui peuvent se transposer dans le monde visuel.

Mais pour répondre à votre question, je pense qu'en fait, une grande partie de ces choses-là, concernant le domaine visuel, ont déjà été réalisées par la géométrie, par la peinture, etc., depuis fort longtemps. L'observation sonore et l'observation visuelle ont des critères fondamentalement différents. Par exemple, il n'y a pas de recul temporel possible sur le son; si vous avez un son de trente secondes (ce qui est long), il a une certaine courbe. Si vous compressez de 10 secondes en le faisant durer 5 secondes, comme on peut le faire numériquement, dans bien des cas, c'est un autre son qu'on obtient.

Question: Mais qu'en est-il du cinéma d'animation, ou du cinéma tout court où justement l'image est elle-même temporelle?

Michel Chion: Dans le cinéma, les formes visuelles sont elles aussi évolutives, mais elles comportent souvent beaucoup d'éléments stables susceptibles de permettre une observation. Ainsi, une voiture qui roule garde ses mêmes proportions en roulant, ou bien ses modifications visibles de forme sont perçues par nous comme créées par la perspective et la distance; ou bien, la variation de vitesse d'un mouvement dans l'image ne fait pas d'un cercle un ovale. Pour le son, c'est plus ambigu. S'il s'agit alors des formes visuelles dans le temps, il y a bien entendu des points communs avec les sons, des profils d'évolution des formes visuelles dans le temps qui appartiennent à ce que j'appelle le «transsensoriel» (voir le Glossaire figurant sur mon site michelchion.com). On ne peut 
toutefois pas totalement décalquer les propriétés du son sur celles de l'image, ni l'inverse. Il existe entre eux des différences profondes.

Par exemple, l'œil explore spatialement une surface. Tout ce que vous voyez dans un film d'animation s'inscrit dans un cadre. Il y a un cadre visible du visuel, mais il n'y a pas de cadre sonore des sons. Le cadre délimite ce qui appartient au film et ce qui ne lui appartient pas, et il structure de façon décisive ce qu'il contient. Ce n'est absolument pas le cas pour le sonore.

Vous entendez dans certaines de mes musiques concrètes, fugitivement, un chien aboyer; même s'il y a peu de probabilités qu'un chien se balade dans la salle de concert, j'aime bien qu'on se pose la question pendant une ou deux secondes, parce que, justement, le son permet de se la poser, du fait qu'il n'y a pas de cadre sonore des sons. On s'en rend compte dans les salles de cinéma lorsqu'une sonnerie de téléphone cellulaire se fait entendre, et qu'on se pose la question pendant quelques secondes de savoir si c'est dans le film ou si c'est un spectateur qui n’a pas coupé son portable. Rien de tel pour le visuel. Le cadre visible du visuel délimite ce qui appartient au film, mais aussi il structure tout ce que l'on voit, et d'ailleurs il arrive à donner une forme plastique aux choses et aux lignes qui se retrouvent dedans: tel élément est plutôt à droite du cadre, plutôt à gauche, plutôt au milieu. Cela produit des effets de composition très prégnants. Si vous changez le cadre dans une photographie, vous changez énormément de choses. Impossible de faire cela pour les sons.

Généralement, cette formule que j’ai mis longtemps à trouver, «il n’y a pas de cadre sonore des sons", rencontre une vive résistance. Plusieurs personnes veulent me prouver qu'il y en a un, sans remarquer qu'ils jouent alors sur des sens très distincts du mot "cadre». C'est curieux comme beaucoup d'intellectuels voudraient que ce qui s'applique au visible s'applique aussi au sonore, et de la même façon. Et aussi, comme ils tiennent absolument à s'accrocher à un mot magique, comme ici le mot "cadre», en en faisant miroiter toutes les significations possibles. Une façon de raisonner, ou plutôt d'user du langage, inspirée selon moi par le discours publicitaire qui ne cesse de nous faire miroiter le nom d'une marque, et de nous dire que, grâce à elle, on a tout à la fois, rien n'est exclu.

Les différences entre image et son sont alors nombreuses, ce qui fait qu'on ne peut pas tout simplement décalquer l'observation d'un domaine sur l'autre, dans les deux sens d'ailleurs. Il faudrait être aussi simple que l'avait fait Schaeffer, qui a su être très simple quand il le fallait. Au niveau du son, celui-ci a pointé une chose que tout le monde savait, mais dont on n'osait pas affirmer l'importance. C'est que l'oreille n'entend pas de la même manière des sons avec une hauteur précise et des sons sans hauteur précise, ce qui pourrait mener soit à deux musiques, soit à deux écoutes parallèles et dissociées dans une même musique. Que l'oreille ne structure pas tout à fait de la même façon les sons toniques et les sons complexes (pour emprunter les termes de Schaeffer), c'est là une différence avec le domaine visuel, où il est plus question de continuum.

Il y a donc un continuum du côté du visuel et un grand discontinuum du côté sonore. Pour ma part, j’assume ce discontinuum. Dans ma musique, je ne dis pas que je veux que tous les sons soient au même niveau. On entend bien 
des voix, donc du langage; on entend bien des notes, des accords; et on entend bien des sons. Il n'y a aucune raison de s'empêcher de faire une musique où se superposent trois ou quatre niveaux d'écoute différents.

À partir du moment où vous constatez que quelqu'un parle, ça se complexifie. C'est très difficile d'observer le son de la voix de quelqu'un qui parle. Vous entendez des phonèmes, même si c'est du latin : requiem eternam. Quand nous entendons re-quiem, e-ter-nam, nous entendons des phonèmes qui appartiennent à la prononciation française du vieux latin. Une grande partie de l'écoute verbale est prédictive, et lorsqu'on écoute de cette façon, il est difficile d'entendre autre chose que la dimension verbale du son. Dans ce contexte, on ne peut pas avoir ce désintéressement, cette abstraction de l'écoute. Je ne cherche même pas à l'obtenir d'ailleurs.

J'ai dit tout à l'heure que je ne voulais pas que l'on connaisse la cause de mes sons. Par contre, je sais que l'auditeur place les sons dans un domaine particulier: musique, bruits, voix. Je ne peux pas empêcher les gens d'entendre une quinte s'il y en a une.

En somme, j'affirme que le visuel et le sonore sont très différents. Ce qui nous cache cette différence, c'est la combinaison de l'un avec l'autre qui donne quelque chose: d'une part, par leur complémentarité, mais, d'autre part, parce que le point commun entre l'image cinématographique, la musique et le son, c'est le rythme et la texture. C'est l'aspect trans-sensoriel dont je vous ai parlé tout à l'heure concernant La Messe de terre. Cet adjectif signifie que ça n'appartient pas nécessairement à la sphère de l'œil, de l'oreille, du toucher, que c'est le rythme.

Nous sommes des êtres de rythme et en même temps nous n'avons pas d'organe de perception du rythme que l'on puisse retirer, localiser, opérer, etc. Mais il est là et il se sert de nos différents sens comme de trous pour faire entrer du rythme. Cela permet au cinéma de faire danser ensemble des images et des sons. On aimerait bien l'idée que le son et l'image se retrouvent, mais je crois que ce sont deux mondes à la fois séparés et magnifiquement complémentaires qui ont en commun, dans les limites que j'ai dites, le temps et le rythme.

\section{L'AVENIR DE L'AUDIOVISUEL ET DE LA MUSIQUE CONCRÈTE}

Question: Est-ce qu'il arrive parfois que vous vous arrêtiez pour penser à ce qu'il reste à faire? De quoi va avoir l'air l'interaction entre la musique et les images dans dix, vingt ou trente ans?

Michel Chion: Je n'en sais rien. Si je comprends bien la première partie de votre question, il s'agit de savoir si l'on peut dire par avance: voici ce qui n'a pas encore été fait, et c'est cela qu'il va falloir faire. Je pense que la création ne fonctionne pas ainsi, de manière logique et linéaire.

Pour l'évolution du cinéma et du multi-media, on verra bien, nul n'est prophète. D'autre part, le cinéma n'est pas une interaction dans l'abstrait entre de la musique et des images. Dans la plupart des cas, il raconte des histoires qui arrivent à des personnages (il est narratif), et il incorpore le texte sous forme orale ou écrite, ou les deux (c'est pourquoi je l'appelle audio-logo-visuel). C'est 
cela qui le définit. Enfin, ses conditions techniques peuvent changer énormément alors que son langage de base reste le même: montage, mixage, découpage, cadrage, etc. C'est ce qui a permis à certains cinéastes qui ont commencé avec la pellicule de poursuivre leur œuvre avec les supports numériques sans presque rien changer de leur style. Nul n'aurait pu prévoir il y a trente ans, à la fois que le numérique s'imposerait si vite dans le cinéma de fiction, et que les cinéastes conserveraient le même style et la même approche, globalement, en passant de la pellicule au numérique: Nurith Bilge Ceylan, Lars von Trier, David Lynch, etc. Il y a trente ans, on prophétisait aussi la disparition à très court terme des salles de cinéma, or, il y en a toujours beaucoup; moins qu'avant certes, mais beaucoup, malgré la VOD, le téléchargement, le DVD, et les dizaines de chaînes de télévision.

L'audio-visuel est un terme abstrait et général: cela peut englober des jeux interactifs, des œuvres qui ne le sont pas, des formules avec multi-écrans, etc. Je ne prétends pas être intéressé par tout ce qu'on fait dans ce domaine - je suis même critique et réservé sur certaines formes artistiques ouvertes et informelles considérées comme plus «modernes» que les autres, telles que les installations vidéo et les installations sonores. Ce n'est pas une question d'hostilité contre les personnes, puisque certains de mes amis en font. Pour ma part, je suis intéressé, captivé, concerné plutôt par les formes fermées dans l'espace et dans le temps, dont relèvent aussi bien la plupart des films de fiction que les œuvres de musique concrète.

Pour ce qui est de la musique concrète, qui n'est pas du tout dans le même schéma économique que le cinéma, elle sera ce que les auteurs, le public, les historiens, en feront. Mais on ne doit pas chercher à l'adapter aux conditions d'écoute les plus courantes aujourd'hui. On ne pouvait pas prévoir en 1990 qu'en 2010 beaucoup de gens pourraient emporter dans leur poche des milliers de morceaux avec eux, pour écouter de la musique dans le métro. Et si on y avait pensé, qu'est-ce que ça aurait donné? On aurait dit qu'il faudrait adapter la musique à ce contexte bruyant, notamment en la compressant, et en évitant des écarts trop forts d'intensité, des formes trop longues et trop complexes, ce que beaucoup de musiciens font maintenant consciemment ou inconsciemment, pour « s'adapter».

Pour ma part, je fais une musique où la forme est souvent longue et comple$\mathrm{xe}$, et où il $\mathrm{y}$ a fréquemment des contrastes entre des sons très piano à certains moments et des sons fortissimo à d'autres. Je le fais ainsi, non pour «résister» à l'esthétique courante, mais parce que je le sens ainsi, je suis seulement mon désir. Donc, mon Requiem ou Le souffle court, ne sont pas des musiques calibrées pour être entendues dans le métro ou en faisant du vélo, même si je sais que les gens ayant acquis ou téléchargé l'œuvre peuvent les écouter comme ils veulent et quand ils veulent. Mais je ne vais rien changer à ma manière de faire ma musique; je fais l'œuvre comme je la sens, et ensuite je formule quelles sont les conditions les meilleures à mon avis pour l'apprécier: dans mon cas, écouter l'œuvre en entier, sans autre activité, et dans un environnement calme permettant la concentration, et ne superposant pas d'autres sons à ceux de l'œuvre. 
Il y a aussi la question d'une esthétique, et de la conscience chez le public et les critiques que cette esthétique est nouvelle. J'ai le sentiment aujourd'hui (que je n'aurais pas pu avoir à l'époque) que mon Requiem de 1973 était en avance sur la plupart des autres musiques, et que curieusement, en 2009, il l'est resté. Ce que certains percevaient comme défaut ou maladresse n'était qu'une façon de faire différente et nouvelle, qui n'est toujours pas vraiment admise, ou qui n'est appréciée que comme élément de mon «style» à moi, et peut-être cette façon d'aborder le genre deviendra-t-elle banale dans vingt ans. Je veux parler de cette approche «médiatiste» que j'ai de la musique concrète, dont je parle dans $L a$ Musique concrète, art des sons fixés. Les compositeurs et les compositrices du futur, et bien sûr les connaisseurs, les historiens, le public, etc. en décideront.

Votre question me tient à cœur, parce que je crois qu'il faut faire comme si ce qu'on créait était destiné à être éternel, parce que sinon on ne s'en sort pas. Et, en même temps, il faut aider la réalité, c'est-à-dire ne pas stipuler des conditions tellement rares pour écouter sa musique que finalement elle ne sera diffusée qu'une seule fois. Il faut faire des compromis dans la diffusion, et aucune dans la conception et la réalisation, si possible. Je vois les choses à long terme, au-delà de ma propre existence. Mes œuvres doivent continuer à vivre après moi; elles sont réalisées dans cet esprit, mais je ne cherche pas non plus à être à l'avant-garde ou dans l'air du temps, ou à anticiper le futur, ou à influencer les jeunes générations. Comme dit Fellini dans son film Roma, il faut faire ce qui vous est «congeniale» (en italien), ce qui vous correspond, ce qu'on sent.

\section{NOTE SUR L'ARTICLE}

Ce texte est une transcription d'un atelier de maître tenu le 10 février 2009 à l'Université de Montréal, dans le cadre des activités du laboratoire «La création sonore : cinéma, arts médiatiques, arts du son» dirigé par Serge Cardinal (transcription : Anne-Marie Leclerc, montage : Pierre Lavoie. Texte revu et complété par Michel Chion en janvier 2012, en plus d'une révision par la rédaction en vue de sa publication). 